STUDI

FRANCESI

\section{Studi Francesi}

Rivista quadrimestrale fondata da Franco Simone

185 (LXII | II) | 2018

OCTAVE MIRBEAU: UNE CONSCIENCE AU TOURNANT

DU SIEECLE - sous la direction de Ida Merello

\title{
ANDREW J. COUNTER, The Amorous Restoration. Love, Sex, and Politics in Early Nineteenth-Century France
}

\section{Clélia Renucci}

\section{(2) OpenEdition \\ Journals}

Édition électronique

URL : https://journals.openedition.org/studifrancesi/13699

DOI : 10.4000/studifrancesi.13699

ISSN : 2421-5856

Éditeur

Rosenberg \& Sellier

\section{Édition imprimée}

Date de publication : 1 août 2018

Pagination : $329-330$

ISSN : 0039-2944

\section{Référence électronique}

Clélia Renucci, « ANDREW J. counter, The Amorous Restoration. Love, Sex, and Politics in Early NineteenthCentury France », Studi Francesi [En ligne], 185 (LXII | II) | 2018, mis en ligne le 01 août 2018, consulté le 16 novembre 2021. URL : http://journals.openedition.org/studifrancesi/13699; DOI : https://doi.org/ 10.4000/studifrancesi.13699

Ce document a été généré automatiquement le 16 novembre 2021.

\section{(c)}

Studi Francesi è distribuita con Licenza Creative Commons Attribuzione - Non commerciale - Non opere derivate 4.0 Internazionale. 


\title{
ANDREW J. COUNTER, The Amorous Restoration. Love, Sex, and Politics in Early Nineteenth-Century France
}

\author{
Clélia Renucci
}

\section{RÉFÉRENCE}

ANDREW J. COUNTER, The Amorous Restoration. Love, Sex, and Politics in Early NineteenthCentury France, Oxford University Press, 2016, 274 pp.

1 L'objet du livre est annoncé clairement: étudier les affinités entre affaires de cœur et affaires d'État dans la France des trente premières années du XIX siècle, et plus précisément de 1815 à 1830 . Entre amour, sexe et mariage d'un côté, et politique de l'autre, l'auteur entend étudier l'intégralité des discours publics sur le sujet dans l'espoir de contribuer à apporter des réponses partielles aux questions que pose l'histoire de la sexualité: comment des idées contradictoires concernant l'amour et le sexe coexistent-elles dans une même société? En quoi ces différentes visions de la moralité sexuelle reflètent-elles les tensions politiques de la communauté? Et enfin, ces tensions trouvent-elles une expression symbolique à travers des objets culturels - la littérature par exemple?

Dans le premier chapitre de son livre, «Impediments. Critiques of Marriage in Restoration France» (pp. 37-76), Andrew COUNTER étudie l'obsession des romanciers du début $\mathrm{du} \mathrm{XIX}^{\mathrm{e}}$ siècle pour les mariages impossibles, qu'ils soient empêchés par des raisons légales, comme la consanguinité, ou morales, comme la différence de croyances ou de classe sociale. Le débat s'élève alors entre des auteurs réformistes comme Étienne de Jouy ou la féministe Hortense Allart qui permettent à leurs héros de surmonter les obstacles et font triompher leur amour, et une littérature plus traditionnelle comme celle de Chateaubriand dans René, de Mme de Duras dans Olivier ou de Charles Nodier dans Thérèse Aubert, qui concluent invariablement à la mort des protagonistes. L'auteur 
entend ainsi montrer comment un topos littéraire comme «l'amour impossible» peut se réinventer, être remotivé pour satisfaire aux besoins culturels et historiques d'une époque.

3 Le deuxième chapitre, intitulé «Good Breeding. Romanticism, Aristocracy, and Reproduction» (pp.77-110), traite de la figure de l'enfant qui, dans les années 1815-1830, devient le symbole de l'épanouissement domestique. Alors que les libéraux reprochent aux ultras de voir l'avenir comme un retour dans le passé, les enfants représentent pour eux la possibilité de l'avènement d'un nouveau monde, la voie la plus sûre vers le salut national. À l'opposé de cette vision positive de la reproduction, la promotion de l'abstinence et la défense du célibat chez un auteur comme Chateaubriand, est, elle aussi, politique: elle permettrait la régulation de la population pour éviter de nouvelles révolutions. L'usage politique de la stérilité par Astolphe de Custine est encore plus retors, étant présentée comme une résistance anti-bourgeoise à la vulgarité et au vice.

4 L'auteur a rappelé dans son introduction l'importance de la censure sous la Restauration. Certains éditeurs de journaux ont décidé de la dévoiler en laissant un espace blanc à l'endroit où l'article aurait dû se trouver pour dénoncer sa suppression imposée. Ce geste politique, par l'absence du contenu interdit, était alors perçu par le lectorat comme aussi, sinon plus subversif que sa présence. Par une judicieuse comparaison, Andrew Counter rapproche cette censure forcée des ellipses sexuelles dans les romans de la Restauration, la plus connue étant peut-être celle du Rouge et le Noir, qui suggère plus qu'elle ne dit. Le chapitre 3, «Gallantries. The Literature of Sexual Worldliness under the Restoration» (pp. 111-141) analyse la littérature galante qui, elle, ne mâche pas ses mots. L'auteur étudie alors la relation entre ces œuvres et le lieu où l'on pouvait se les procurer: le Palais-Royal, que Balzac décrit si précisément dans Illusions perdues et qui devient le symbole du rejet de tout moralisme sexuel. Cette littérature, reconfiguration du libertinage, est en fait politique, et l'objectif est de lutter contre les contraintes nouvelles imposées par le siècle.

5 Le chapitre 4, "Olivier in the Closet: Gossip, Scandal, and the Novel in the 1820s» (pp. 142-173), s'intéresse au cas d'Olivier, roman tiré de l'histoire d'Astolphe de Custine. Le scandale provoqué par son affaire - alors que Custine se rendait à un rendez-vous galant avec un jeune soldat, il fut battu sur le chemin par d'autres soldats - a anéanti sa réputation. Ce n'est pas l'aventure en soi qui intéresse Andrew Counter, mais les réactions qu'elle a provoquées; trois romans au moins furent publiés, dont celui de Mme de Duras. À travers l'étude de ce texte, l'auteur montre que la stratégie du secret, du roman à clef, le rôle de l'omission, ne relève pas que d'une simple protection: elle est la manifestation d'une résistance à la censure et à l'oppression que le gouvernement faisait alors peser sur les écrits.

Le chapitre 5, "The Sexual Monster: Celibacy and the Anticlerical Imagination» (pp. 174-207), débute par le récit du viol et du meurtre d'une paroissienne par le prêtre Antoine Mingrat le 8 mai 1822, condamné à mort le 9 décembre. Ayant réussi à s'échapper en Sardaigne, le gouvernement n'a pas imposé son extradition et celui-ci n'a finalement jamais subi sa peine. Un scandale s'ensuivit et ouvrit des questions politiques majeures. Cette affaire devint un thème privilégié du discours anticlérical et la conspiration gouvernementale présumée fut vivement critiquée dans les cercles libéraux. Andrew Counter s'intéresse ici à l'instrumentalisation politique de l'idée de normalité sexuelle et cherche à mettre en évidence le paradoxe central du cas Mingrat: 
son crime est à la fois traité comme quelque chose de monstrueux et de naturel l'érotomanie des prêtres, par leur célibat contraint, est perçue alors comme consubstantielle de leur état - comme excessivement choquant et nécessairement logique - les lecteurs de la Restauration étant culturellement conditionnés pour voir dans la violence masculine une expression de la sexualité mâle.

7 On ne peut imaginer deux auteurs plus opposés, une amitié plus contradictoire que celle dont il est question dans le chapitre 6 de cet ouvrage, «1830: The End of an Eros? Nodier and Fourier on the History of Sexuality» (pp. 208-239), celle de Charles Nodier, fervent défenseur du régime Bourbon, et celle de Charles Fourier, radical, fantasque et toujours outsider. Et pourtant, l'auteur s'attache à saisir les quelques thèmes qui les rapprochent: l'inconstance historique de la moralité sexuelle d'abord, mais aussi, à propos de l'amour et du sexe, les liens entre les mœurs et les changements politiques. Plus précisément, Andrew Counter va s'intéresser au cas de l'inceste, comme moyen d'enquêter sur la nature de la morale sexuelle en général. Les deux auteurs suggèrent que les évolutions du tabou de l'inceste prouvent l'absurdité de toute morale concernant le sexe et préfigurent la chute des normes sexuelles dans le futur.

8 Pour finir, disons un mot des références à la queer theory dans ce livre. Si l'auteur démontre bien sûr sa connaissance des débats et des recherches en cours, il rappelle que cet ouvrage n'a pas pour objet d'y prendre part. Le chapitre 1 - où Andrew Counter évoque la question du mariage dans les années 1820 - et le chapitre 6 - où sont étudiées les critiques des normes sexuelles par Nodier et Fourier - anticipent les débats de la fin $\mathrm{du} \mathrm{xx}^{\mathrm{e}}$ siècle en montrant que les mœurs sexuelles sont changeantes et dépendent essentiellement de l'époque dans laquelle on se situe. Et si, bien sûr, la question du mariage entre personnes du même sexe n'est pas posée au début du XIX ${ }^{e}$ siècle, Nodier et Fourier s'intéressant plutôt à celle de l'inceste, ces discours peuvent servir à éclairer notre présent. On ne peut nier qu'un travail sur le sexe dans l'histoire évoque nécessairement les interrogations qui concernent le sexe dans le présent, et l'auteur espère que, par la présentation des excentricités de la Restauration, ce livre pourra créer des ponts provocateurs et productifs entre les théories d'hier et celles d'aujourd'hui sur l'amour, le sexe et la politique. Plus que des analogies, Andrew Counter cherche à apporter des réponses généalogiques à nos débats contemporains. 